ALGORITHM and CHARTS TO CALCULATE and MODIFY TUNES and CHROMATICITY in the AGS BOOSTER, PROTON CASE

BOOSTER TECHNICAL NOTE

NO. 179

A. LUCCIO

OCTOBER 17, 1990

ALTERNATING GRADIENT SYNCHROTRON DEPARTMENT BROOKHAVEN NATIONAL LABORATORY UPTON, NEW YORK 11973 


\section{ALGORITHM AND CHARTS TO CALCULATE AND MODIFY TUNES AND \\ CHROMATICITY IN THE AGS BOOSTER. PROTON CASE.}

\section{A.LUCCIO}

\section{Introduction}

Tune and chromaticity are a function of the quadrupole and sextupole setting. During commission and operation it is desirable to have ready an algorithm, charts and tables to calculate the currents in the quadrupoles and sextupoles that will yield specific values for these parameters.

The tune $v$ is in good approximation a linear function of the quadrupole current $\mathfrak{S}$ over a rather wide range of values. Then, it is possible to calculate from the model a transformation matrix between currents and values of $v$ that does not appreciably vary from point to point over a tune chart. The chromaticity $\xi$ is not very linear with $\mathfrak{I Q}$, but still a matrix can be calculated in the immediate surrounding of a given point.

The tune does not depend on the sextupoles, while the chromaticity is a good linear function of the currents in the sextupoles $\mathfrak{I}^{\mathrm{s}}$. From the model, transformation matrices between $\xi$ and $\mathfrak{I}^{\mathrm{s}} \mathrm{can}$ be calculated.

In the AGS Booster the quadrupoles have a main set of coils which carry the same currents as the machine dipoles and a set of tune coils. The quadrupoles have been recently thoroughly measured ${ }^{1}$ and the calculations in this report are based on these most recent values. For the dipole parameters we used recent measured -although not yet published- values ${ }^{2}$. For the sextupoles, the values given in the Manual $^{3}$ were used.

This report is limited to the acceleration cycle of protons. It follows and updates previous studies on the same matter ${ }^{4}$

1 E.Bleser. AGS Booster Technical Reports \#174 and \#176, September 1990

2 R.Thern. Private communication

3 Booster Design Manual, Revision 1, October 1988, p. 1-12

4 S.Y.Lee. AGS Booster Technical Reports \#168 and \#169, June 1990 


\section{The bare machine.}

With the model MAD 5 and coefficients and magnetic effective lengths from the measurements, tables of the tune and chromaticity with a variety of quadrupole and sextupole settings were calculated. The calculations were performed in the limit of a zero current beam and the effects of eddy currents were not included. The tune of the "bare" machine, i.e. when the quads carry only the main current, vary very little along the accelerating cycle. Results are in Table I, with the relation between magnetic rigidity and proton kinetic energy $E_{k}$

$$
B \rho=\frac{\sqrt{E_{k}\left(E_{k}+2 E_{0}\right)}}{0.3}
$$

$B \rho$ is in tesla-m, $E_{k}$ and $E_{0}$ in GeV, $\rho=13.8656 m$ and $E_{0}=0.938280 \mathrm{GeV}$. For protons

$$
\begin{aligned}
& B \rho=2.14816 \mathrm{~T}-\mathrm{m}, \text { at injection }\left(\mathrm{E}_{\mathrm{k}}=0.200 \mathrm{GeV}\right) \\
& \mathrm{B} \rho=7.50174 \mathrm{~T}-\mathrm{m}, \text { at extraction }\left(\mathrm{E}_{\mathrm{k}}=1.500 \mathrm{GeV}\right)
\end{aligned}
$$

The quadrupoles' gradient is defined as

$$
K_{1}=\frac{1}{B \rho} \frac{\partial B}{\partial r} \quad ; \quad B_{1}=\frac{\partial B}{\partial r}
$$

where $B_{1}$ is the quantity in Ref ${ }^{1}$. The sextupoles' gradient is defined as

$$
K_{2}=\frac{1}{B \rho} \frac{\partial^{2} B}{\partial r^{2}}=\frac{B^{\prime \prime}}{B \rho}
$$

with the "Manual"3 value of B" of $8.8 \mathrm{~T} / \mathrm{m}^{2}$. We define the chromaticity as

$$
\xi=\frac{\Delta v / v}{\Delta p / p}
$$

5 F.Ch.Iselin and J.Niederer. MAD. CERN/LEP-TH-87/33. Geneva, April 1987 
Table I.

AGS Booster: bare machine.

Dipole field, proton kinetic energy, tune and chromaticity as a function of the dipole main current

\begin{tabular}{|cccccccc|}
\hline $\mathrm{I}^{\mathrm{D}}[\mathrm{A}]$ & $\mathrm{B}^{\mathrm{D}}[\mathrm{T}]$ & $\mathrm{E}_{\mathrm{k}}[\mathrm{GeV}]$ & $\mathrm{K}_{1}\left[\mathrm{~m}^{-2}\right]$ & $v_{\mathrm{x}}$ & $v_{\mathrm{y}}$ & $\xi_{\mathrm{x}}$ & $\xi_{\mathrm{y}}$ \\
\hline 600 & 0.14593 & 0.17924 & 0.54888 & 4.649 & 4.568 & -0.9920 & -1.0727 \\
637.01 & 0.15493 & 0.200 & 0.54889 & 4.649 & 4.568 & & \\
800 & 0.19456 & 0.30081 & 0.54900 & 4.650 & 4.569 & -0.9922 & -1.0729 \\
1,000 & 0.24321 & 0.44152 & 0.54906 & 4.650 & 4.570 & -0.9921 & -1.0729 \\
1,200 & 0.29186 & 0.59608 & 0.54913 & 4.651 & 4.570 & -0.9924 & -1.0730 \\
1,400 & 0.34049 & 0.76066 & 0.54923 & 4.652 & 4.571 & -0.9925 & -1.0731 \\
1,600 & 0.38910 & 0.93255 & 0.54936 & 4.653 & 4.572 & -0.9926 & -1.0732 \\
1,800 & 0.43768 & 1.1099 & 0.54952 & 4.654 & 4.574 & -0.9928 & -1.0734 \\
2,000 & 0.48625 & 1.2914 & 0.54968 & 4.656 & 4.575 & -0.9930 & -1.0735 \\
2,200 & 0.53479 & 1.4760 & 0.54976 & 4.656 & 4.576 & -0.9931 & -1.0736 \\
$2,225.7$ & 0.54103 & 1.500 & 0.54978 & 4.657 & 4.576 & & \\
2,400 & 0.58336 & 1.6634 & 0.54982 & 4.657 & 4.577 & -0.9932 & -1.0737 \\
\hline
\end{tabular}

With MAD we calculate in a loop the tune $\left(v_{x}, v_{y}\right)$ and the chromaticity $\left(\xi_{x}, \xi_{y}\right)$ for a set of values of the currents in the quadrupoles $\left(\left|q_{x},\right| q_{y}\right)$ and in the sextupoles $\left(\left|s_{x},\right| s_{y}\right)$. In each point of a $v$ $=\left(v_{x}, v_{y}\right)$ plane -and similarly of a $\xi=\left(\xi_{x}, \xi_{y}\right)$ plane- we can define the following linear transformations

$$
\mathfrak{I}^{q}=\mathbf{Q} \cdot v \quad ; \quad \mathfrak{I}^{q}=\mathbf{X}^{\mathrm{q}} \cdot \boldsymbol{\xi} \quad ; \quad \mathfrak{S}^{\mathrm{s}}=\mathbf{X}^{\mathrm{s}} \cdot \boldsymbol{\xi}
$$

with $\mathbf{Q}=\mathbf{Q}$ and $\mathbf{X}=\mathbf{X} 2 \times 2$ matrices, $v$ and $\xi$ vectors, and $\mathfrak{I}^{q}$ and $\mathfrak{I}^{\mathcal{S}}$ the vector of currents in the quadrupoles and sextupoles. The matrices $\mathbf{Q}$ and $\mathbf{X}$ are in general a function of the point in the planes. A set of matrices is calculated and stored in a file: then the values of currents for a given value of the tunes and/or the chromaticity can be found by interpolation.

\section{Tune and chromaticity charts (protons: injection, extraction)}

Let us set the $B \rho$ value at injection. By varying the currents in the tune trim coil in both families of quadrupoles, the charts of figures 1, 2, 3 and 4 are obtained. 
Figure 1 shows a tune chart for protons at injection, for a wide range of excitation in the horizontal (focusing) quadrupoles -marked by the upper case letters- and in the vertical quadrupoles -lower case letters- The values of the quadrupole currents are given in Table II, the available range of the tune trim coil current is $\pm 500 \mathrm{~A}$. The bare machine tune at injection is also shown in the figure.

The chromaticity is also dependent from the quadrupole setting. Figure 2 shows a chromaticity chart for protons at injection, for the same currents as in figure 1 and Table II. The bare machine chromaticity at injection is also shown in the figure.

In table II, Jequiv is the current that in the main coil only would have produced the required

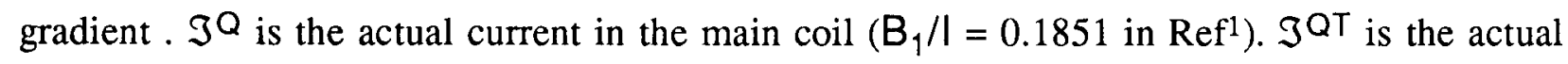
current in the tune trim coil $\left(B_{1} / l=3.70610^{-4} \mathrm{~T} / \mathrm{m}-\mathrm{A}\right.$ in $\left.\operatorname{Ref}^{1}\right)$.

\section{Table II}

Protons. Injection.

Currents in the quadrupoles that produce the tune in figure 1 and chromaticity in figure 3

\begin{tabular}{|cccc|}
\hline & $\begin{array}{c}\text { Jequiv } \\
{[\mathrm{A}]}\end{array}$ & $\begin{array}{c}\text { JQ } \\
{[\mathrm{A}, \text { main coil] }}\end{array}$ & $\begin{array}{c}\mathfrak{J}^{\mathrm{QT}} \\
{[\mathrm{A}, \text { tune trim coil] }}\end{array}$ \\
\hline $\mathrm{A}, \mathrm{a}$ & 540 & 637.010 & -484.57 \\
$\mathrm{~B}, \mathrm{~b}$ & 560 & & -384.67 \\
$\mathrm{C}, \mathrm{c}$ & 580 & & -284.77 \\
$\mathrm{D}, \mathrm{d}$ & 600 & & -184.87 \\
$\mathrm{E}, \mathrm{e}$ & 620 & & -84.96 \\
$\mathrm{~F}, \mathrm{f}$ & 640 & & 14.93 \\
$\mathrm{G}, \mathrm{g}$ & 660 & & 114.83 \\
$\mathrm{H}, \mathrm{h}$ & 680 & & 214.72 \\
$\mathrm{I}, \mathrm{i}$ & 700 & & 314.64 \\
$\mathrm{~J}, \mathrm{j}$ & 720 & & 414.54 \\
\hline
\end{tabular}

Figure 3 is simply a blow-up of the central part of figure 1, where the dependence of the tune from the currents is more linear. The values of the quadrupole currents are given in table III. 
Similarly, figure 4 is a blow-up of the central part of figure 3, with values in table III. The dependence of the chromaticity from quadrupole setting is not very linear, but its range of variations is not wide.

Table III

Protons. Injection.

Currents in the quadrupoles that produce the tune in figures 3 and the chromaticity in figure 4.

\begin{tabular}{|cccc|}
\hline & $\begin{array}{c}\text { Iequiv }_{\text {equ }} \\
{[\mathrm{A}]}\end{array}$ & $\begin{array}{c}\mathrm{IQ} \\
{[\mathrm{A}, \text { main coil }]}\end{array}$ & $\begin{array}{c}\text { IQT } \\
{[\mathrm{A}, \text { tune trim coil }]}\end{array}$ \\
\hline $\mathrm{A}, \mathrm{a}$ & 620 & 637.010 & -84.96 \\
$\mathrm{~B}, \mathrm{~b}$ & 630 & & -35.01 \\
$\mathrm{C}, \mathrm{c}$ & 640 & & 14.93 \\
$\mathrm{D}, \mathrm{d}$ & 650 & & 64.88 \\
$\mathrm{E}, \mathrm{e}$ & 660 & & 114.83 \\
$\mathrm{~F}, \mathrm{f}$ & 670 & & 164.77 \\
$\mathrm{G}, \mathrm{g}$ & 680 & & 214.72 \\
$\mathrm{H}, \mathrm{h}$ & 690 & & 264.66 \\
\hline
\end{tabular}

As an example, from figure 3 , the value $v_{x} \approx v_{y} \approx 4.8$ is obtained with $\mathrm{I}_{\text {eq }} \approx(660,-660) \mathrm{A}$, or $\mathrm{I}^{\mathrm{Q}}=$ $637.01 \mathrm{~A}$ in the main coils and $\mathrm{PT}=(115.47,104.10) \mathrm{A}$ in the tune coils, horizontal and vertical family, respectively.

The Chromaticity is best controlled by the currents in both families of sextupoles. Results for protons at injection are shown in figure 5 and table IV.

In the figure, we show a range of sextupole $\mathrm{K}_{2}$ values from $\pm 2 \mathrm{~m}^{-3}$. With the standard value of $\mathrm{B}^{\prime \prime}$ of $8.8 \mathrm{~T} / \mathrm{m}^{2}$, the allowed range at injection is $\approx \pm 4.4 \mathrm{~m}^{-3}$. (Since the Booster power supplies for the sextupoles are unipolar, to change the sign of the currents one should physically change connections). The figure shows three regions of values, separated by discontinuities where $\xi_{x}$ or $\xi_{y}$ change sign. The values of $\mathrm{K}\left(\equiv \mathrm{K}_{2}\right)$ for each region are given in the table. 
Similarly, figure 4 is a blow-up of the central part of figure 3, with values in table III. The dependence of the chromaticity from quadrupole sctting is not very linear, but its range of variations is not wide.

Table III

Protons. Injection.

Currents in the quadrupoles that produce the tune in figures 3 and the chromaticity in figure 4 .

\begin{tabular}{|cccc|}
\hline & $\begin{array}{c}\text { Jequiv } \\
{[\mathrm{A}]}\end{array}$ & $\begin{array}{c}\mathfrak{J}^{\mathrm{Q}} \\
{[\mathrm{A}, \text { main coil] }}\end{array}$ & $\begin{array}{c}\mathfrak{J Q T}^{\mathrm{QT}} \\
{[\mathrm{A}, \text { tune trim coil] }}\end{array}$ \\
\hline A, a & 620 & 637.010 & -84.96 \\
B, b & 630 & & -35.01 \\
C, c & 640 & & 14.93 \\
D, d & 650 & & 64.88 \\
E, e & 660 & & 114.83 \\
F, f & 670 & & 164.77 \\
G, g & 680 & & 214.72 \\
H, h & 690 & & 264.66 \\
\hline
\end{tabular}

As an example, from figure 3 , the value $v_{x} \approx v_{y} \approx 4.8$ is obtained with Jequiv $\approx(660,-660) \mathrm{A}$, or $\mathfrak{I}^{\mathrm{Q}}=637.01 \mathrm{~A}$ in the main coils and $\mathfrak{J}^{\mathrm{QT}}=(115.47,104.10) \mathrm{A}$ in the tune coils, horizontal and vertical family, respectively.

The Chromaticity is best controlled by the currents in both families of sextupoles. Results for protons at injection are shown in figure 5 and table IV.

In the figure, we show a range of sextupole $\mathrm{K}_{2}$ values from $\pm 2.0 \mathrm{~m}^{-3}$. With the standard value of $\mathrm{B}^{\prime \prime}$ of $8.8 \mathrm{~T} / \mathrm{m}^{2}$, the allowed range at injection is $\approx \pm 4.4 \mathrm{~m}^{-3}$. (Since the Booster power supplies for the sextupoles are unipolar, to change the sign of the currents one should physically change connections). The figure shows three regions of values, separated by discontinuities where $\xi_{x}$ or $\xi_{y}$ change sign. The values of $K\left(\equiv K_{2}\right)$ for each region are given in the table. 
Table IV

Protons. Injection.

Currents in the sextupoles that produce the chromaticity in figure 5.

\begin{tabular}{|cccc|}
\hline $\mathrm{K}_{2, y}$ & $\begin{array}{c}\mathrm{K}_{2, \mathrm{x}} \\
(\mathrm{C})\end{array}$ & $\begin{array}{c}\mathrm{K}_{2, \mathrm{x}} \\
(\mathrm{A})\end{array}$ & $\begin{array}{c}\mathrm{K}_{2, \mathrm{x}} \\
(\mathrm{B})\end{array}$ \\
\hline-2.0 & & $-2.0 \rightarrow 0.5$ & $1.0 \rightarrow 2.0$ \\
-1.5 & & $-2.0 \rightarrow 0.5$ & $1.0 \rightarrow 2.0$ \\
-1.0 & & $-2.0 \rightarrow 0.5$ & $1.0 \rightarrow 2.0$ \\
-0.5 & & $-2.0 \rightarrow 1.0$ & $1.5 \rightarrow 2.0$ \\
+0.0 & & $-2.0 \rightarrow 1.0$ & $1.5 \rightarrow 2.0$ \\
+0.5 & & $-2.0 \rightarrow 1.0$ & $1.5 \rightarrow 2.0$ \\
+1.0 & $-2.0 \rightarrow-1.5$ & $-1.0 \rightarrow 1.0$ & $1.5 \rightarrow 2.0$ \\
+1.5 & $-2.0 \rightarrow-0.5$ & $+0.0 \rightarrow 1.5$ & 2.0 \\
+2.0 & $-2.0 \rightarrow+0.5$ & $+1.0 \rightarrow 1.5$ & 2.0 \\
\hline
\end{tabular}

Figure 6,7 and 8 propose similar charts at extraction (1.5 GeV, protons).

Figure 6 is a tune chart for the full range ( $\pm 500 \mathrm{~A}$ ) of quadrupole trim current. Upper case letters mark lines of constant $\mathfrak{I}^{Q_{x}}$ and lower case lines of constant $\mathfrak{I}^{Q_{y}}$. The values are in table $\mathrm{V}$.

Figure 7 and table $\mathrm{V}$ give values of the chromaticity for full quadrupole excitation.

Figure 8 shows a chart of the chromaticity, within the full range of values of the sextupole gradient $\mathrm{K}_{2}$, which is $\pm 1.17 \mathrm{~m}^{-3}$ at extraction. There were discontinuities in the chromaticity chart and three regions of values appeared -like described in connection with figure 5. figure 8 only shows the "main" region.

\section{Algorithm}

We have prepared a script, TUNES, and a set of algoritms that allows the operator to find the currents in the quadrupoles and sextupole to obtain a given tune and chromaticity. 
Table V

Protons. Extraction.

Currents in the quadrupoles that produce the tune in figures 6 and the chromaticity in figure 7.

\begin{tabular}{|cccc|}
\hline & $\begin{array}{c}\text { Jequiv } \\
{[\mathrm{A}]}\end{array}$ & $\begin{array}{c}\mathfrak{J}^{\mathrm{Q}} \\
{[\mathrm{A}, \text { main coil] }}\end{array}$ & $\begin{array}{c}\mathfrak{J}^{\mathrm{QT}} \\
{[\mathrm{A}, \text { tune trim coil] }}\end{array}$ \\
\hline $\mathrm{A}, \mathrm{a}$ & 2125 & $2,225.70$ & -503.00 \\
$\mathrm{~B}, \mathrm{~b}$ & 2150 & & -378.12 \\
$\mathrm{C}, \mathrm{C}$ & 2175 & & -253.25 \\
$\mathrm{D}, \mathrm{d}$ & 2200 & & -128.37 \\
$\mathrm{E}, \mathrm{e}$ & 2225 & & -3.50 \\
$\mathrm{~F}, \mathrm{f}$ & 2250 & & 121.38 \\
$\mathrm{G}, \mathrm{g}$ & 2275 & & 246.25 \\
$\mathrm{H}, \mathrm{h}$ & 2300 & & 371.13 \\
$\mathrm{I}, \mathrm{i}$ & 2325 & & 496.00 \\
\hline
\end{tabular}

Step (i) is to run MAD and create a set of files -called qxi_matrix.data- storing the transform matrices $\mathbf{Q}, \mathbf{X} \mathbf{9}$ and $\mathbf{X} \boldsymbol{S}$ over the widest possible range of tune and chromaticity allowed by the magnet' power supplies. Given the geometry of the machine, in principle we should prepare matrix files for each gauss-clock time during the acceleration, since the magnet parameters vary with the field, due to saturation effects. In practice, we will prepare only a few -say six-for notable times, including injection and extraction. The files are permanently stored -probably in the database- and need to be recalculated only if the basic machine is changed.

Step (ii): the operator is queried to enter the sought values of the tune(s) and the quadrupole trim currents are calculated and dispalyed (perhaps stored in a file for subsequent use). The algorithm for this is straightforward: since the $\mathbf{Q}$ matrix is almost constant over the full range of tunes, the matrix used for the calculation is the one corresponding to the tune point closest to the required tune. At this stage, also the chromaticity is calculated with the matrix $\mathbf{X q}$ : call it $\xi^{(0)}$.

Step (iii): the operator is queried for a value of $\xi$. The procedure to calculate the chromaticity, starting from a value $\xi^{(0)}$, would in principle require the help of MAD to recalculate matrices $\mathbf{X}^{S}$ based on the un-bare machine. Fortunately, the variation of $\xi$ due to the quadrupoles is small (e.g. compare the ranges in figures 7 and 8 ) and a linear perturbation algorithm is acceptable. 
The program operates in this way: using the matrix $\mathbf{X}$ calculated at (near) $\xi^{(0)}$, it finds the value of the current (2-dimensional vector) in the sextupoles which would give $\xi^{(0)}$ from the bare machine; call it $\mathfrak{I}^{\mathfrak{S}(0)}$. Then, it calculates the current that would give $\xi$ from the bare machine; call it $\mathfrak{I}^{S(1)}$. The resulting current to be applied to the sextupoles is therefore $\mathfrak{I}^{\mathfrak{S}}=\mathfrak{I}^{\mathfrak{S}(1)}-\mathfrak{I}^{\mathfrak{S}(0)}$. 


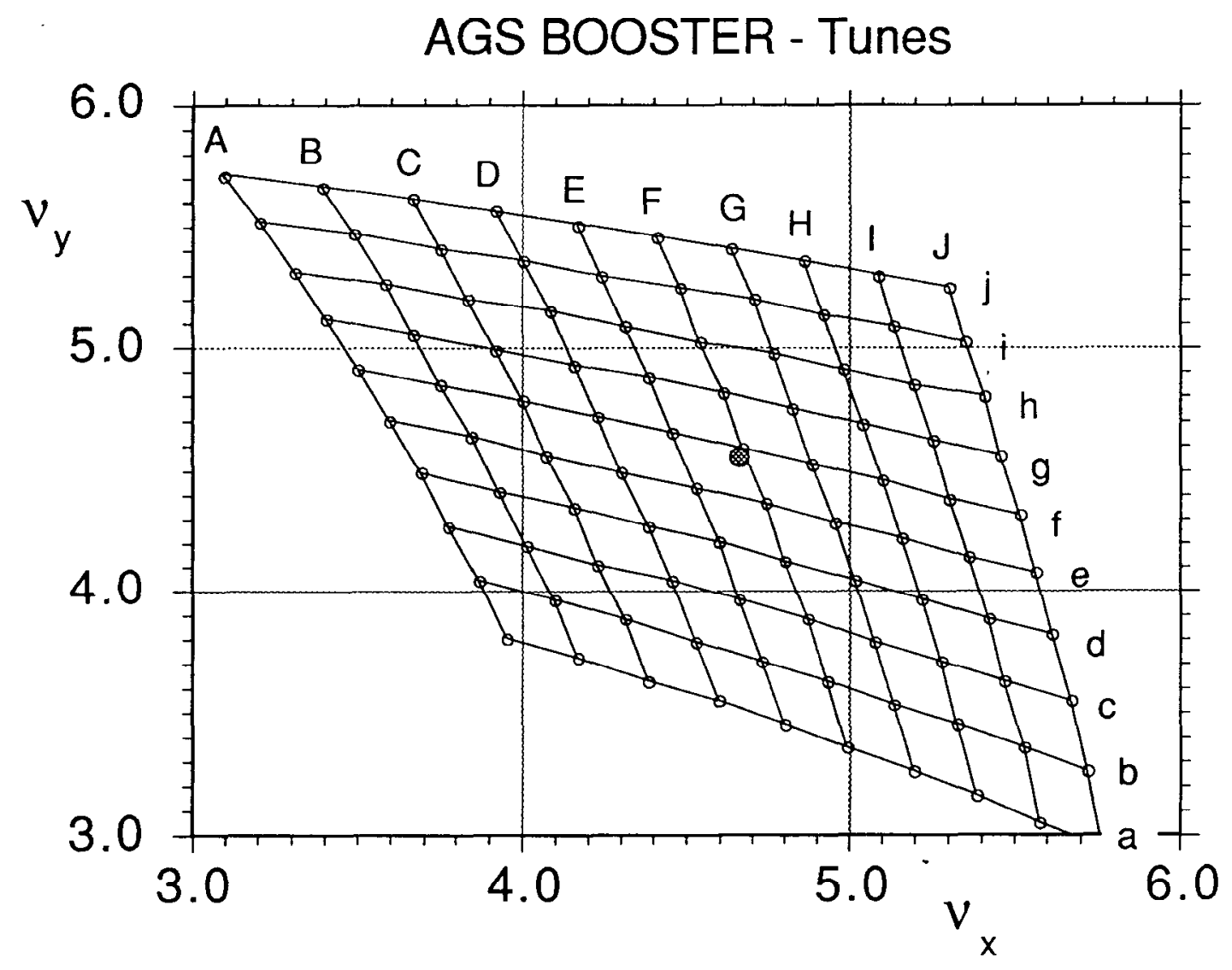

Fig. 1. Tune chart for protons at injection, by varying quadrupoles (table II). The bare machine tune at injection is marked by the gray dot. 


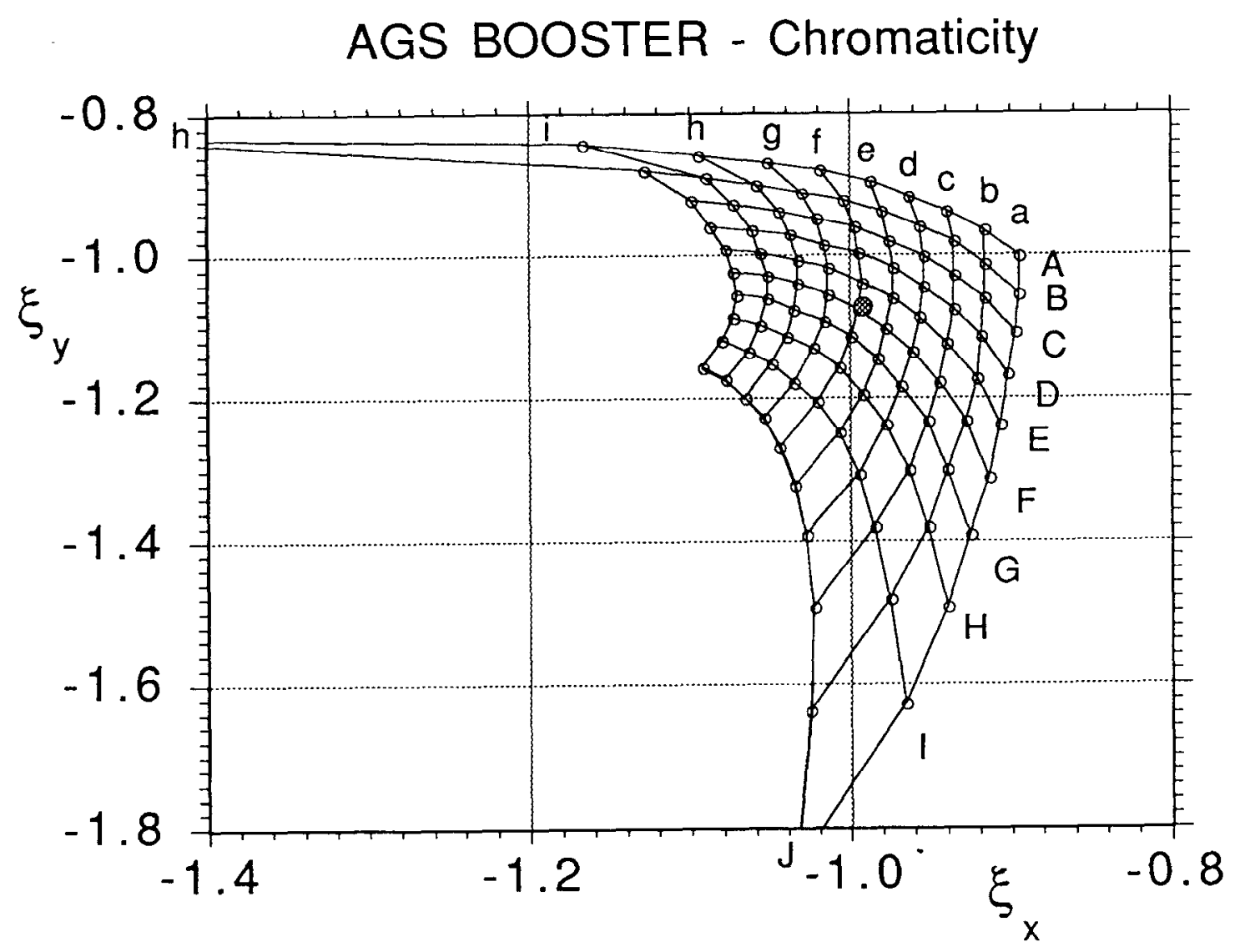

Fig. 2. Chromaticity for protons at injection, by varying quadrupoles (table II). The bare machine value at injection is marked by the gray dot. 


\section{AGS BOOSTER - Tunes}

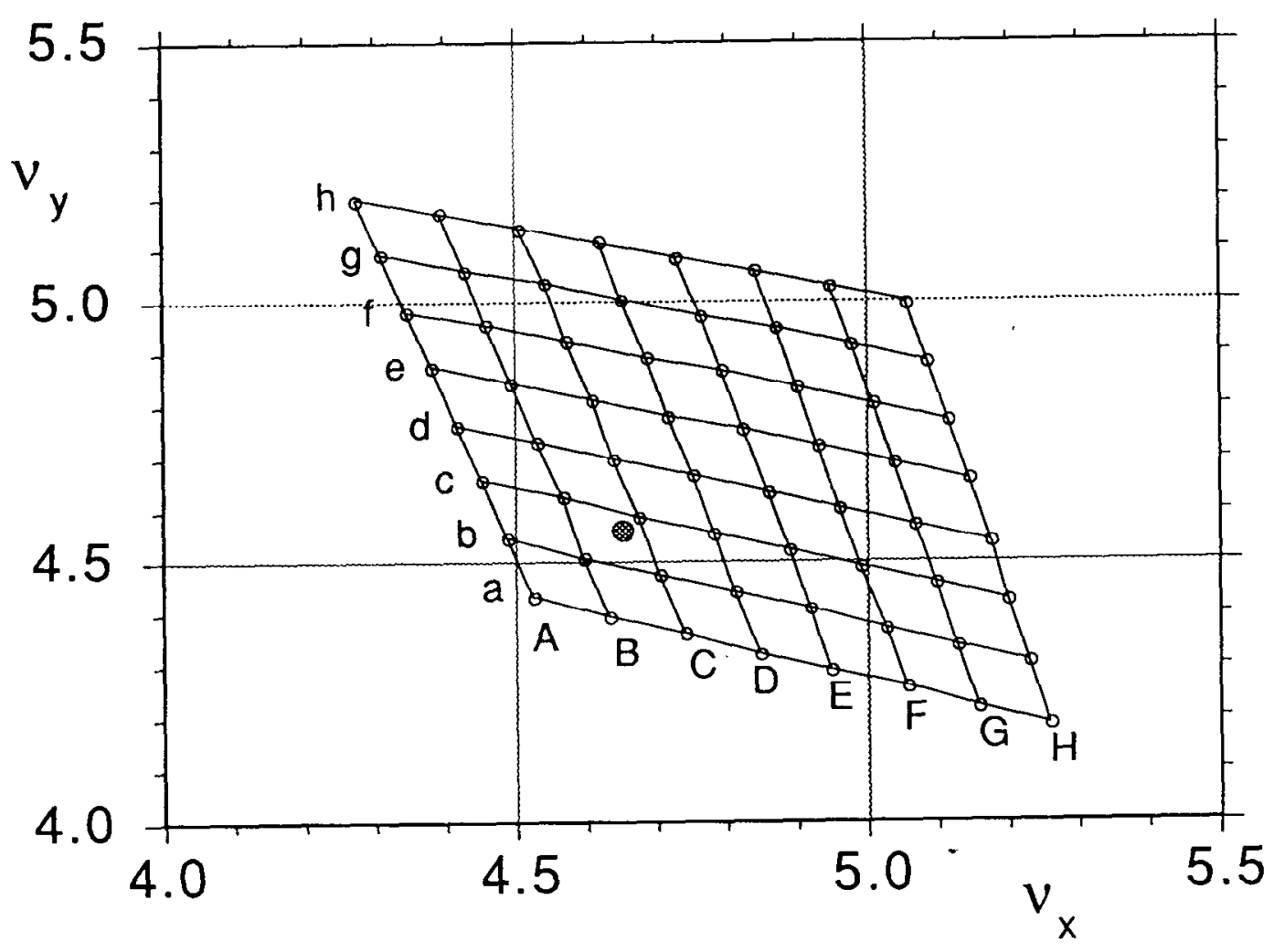

Fig. 3. Tune chart for protons at injection, by varying quadrupoles (table III). Blow-up of figure 1. 


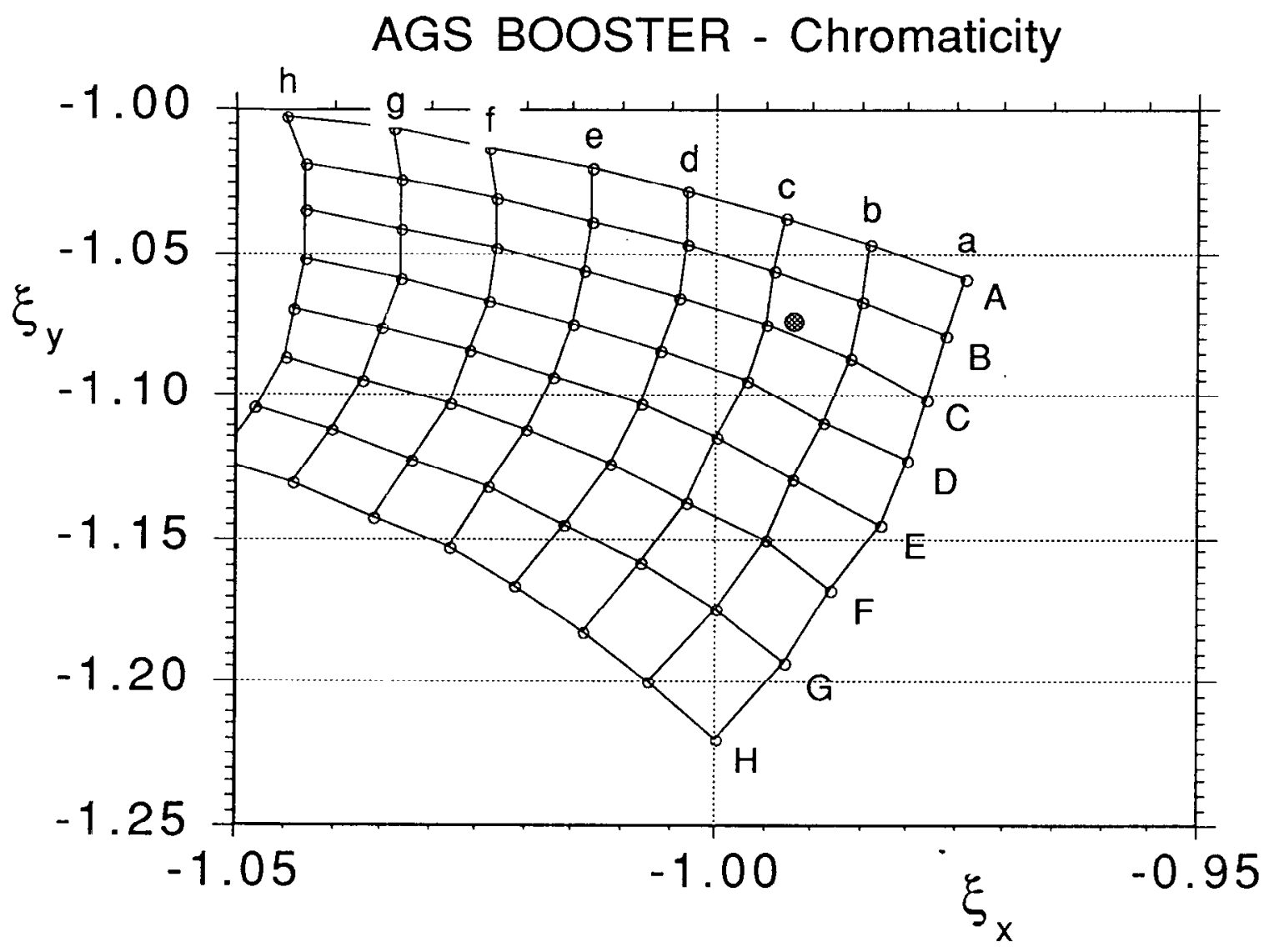

Fig. 4. Chromaticity for protons at injection, by varying quadrupoles (table III). Blow-up of figure 2. 


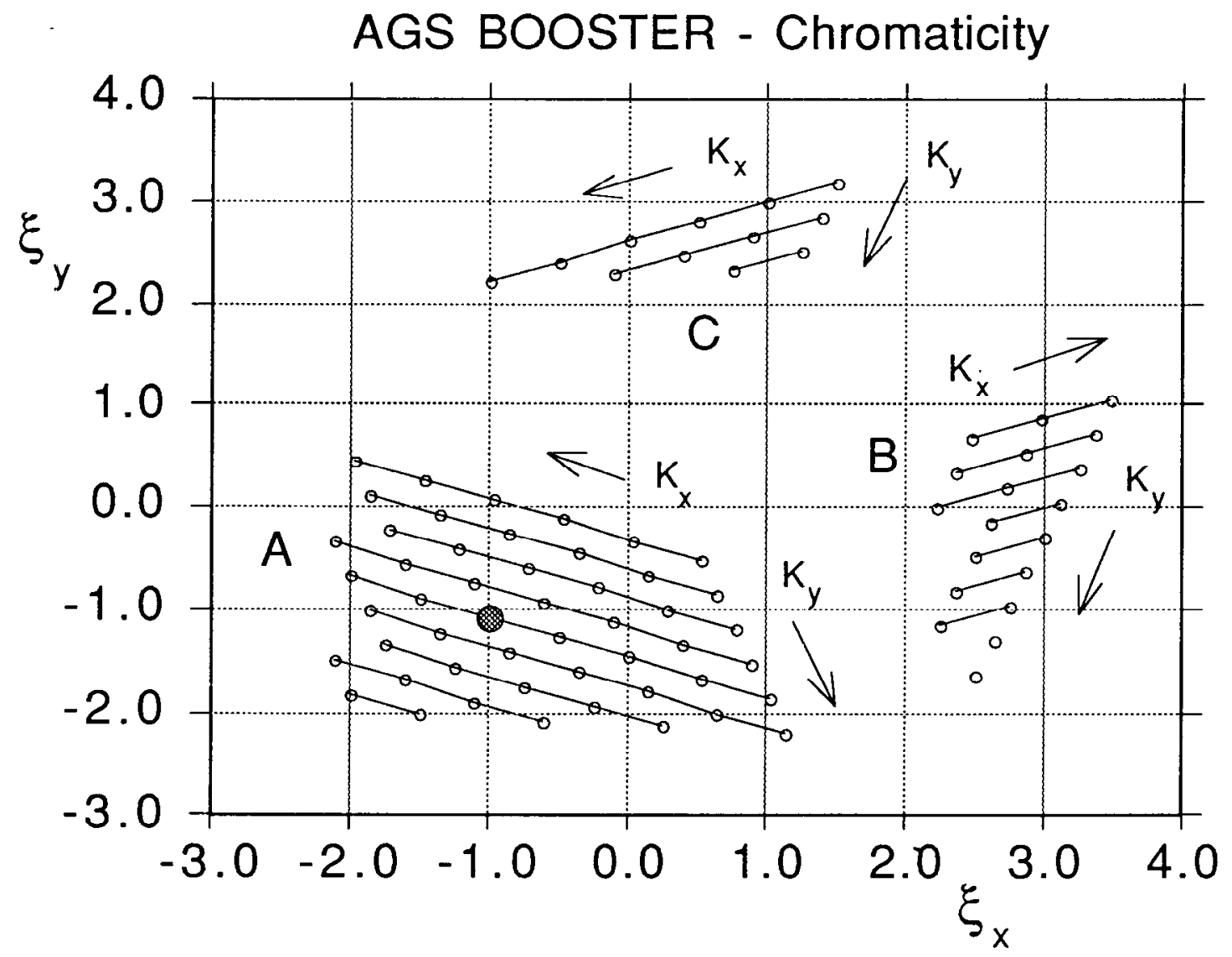

Fig. 5. Chromaticity for protons at injection, by varying sextupoles (table IV). Three regions of values appear. The bare machine value at injection is marked by the gray dot. 


\section{AGS BOOSTER - Tunes}

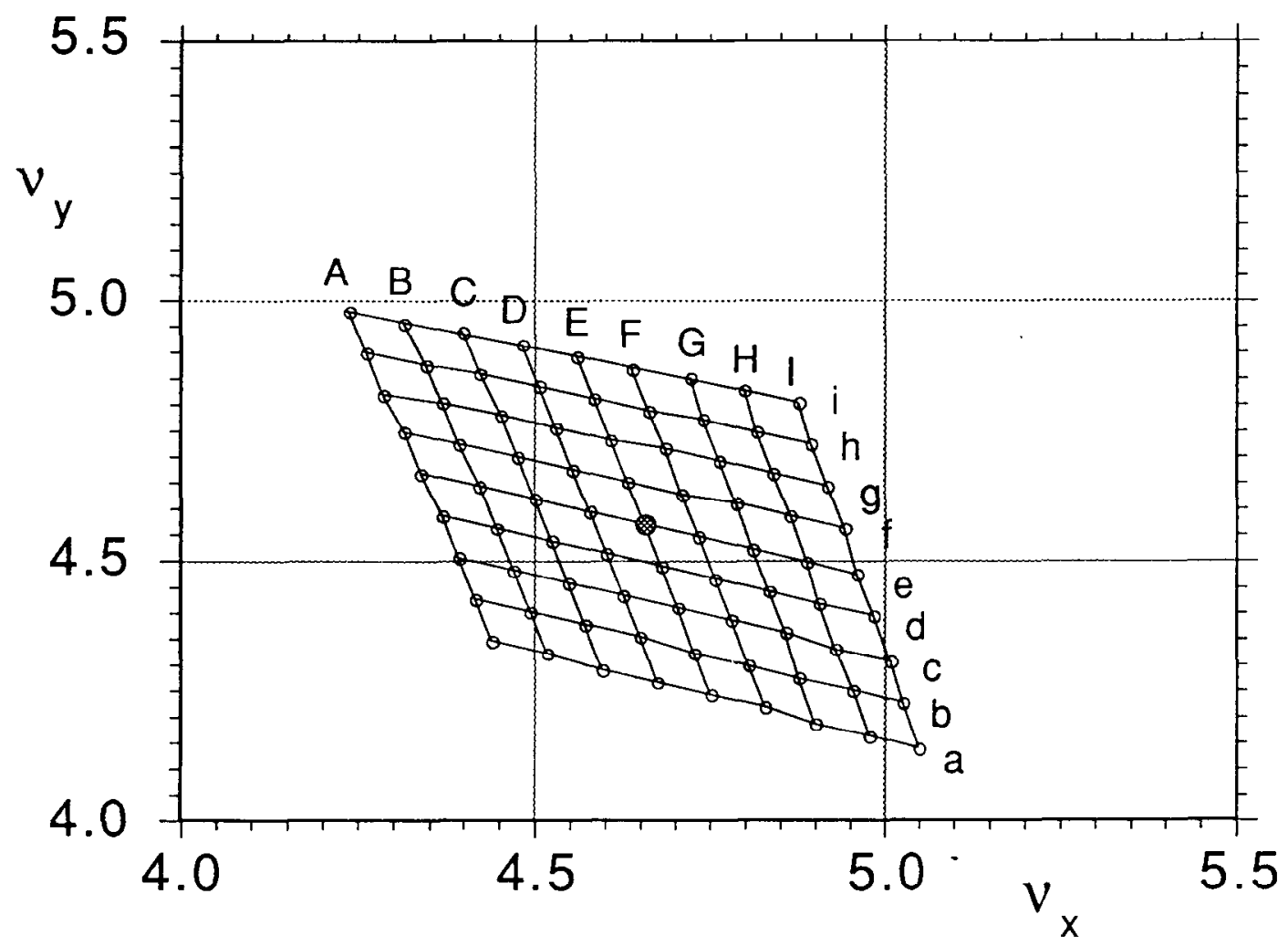

Fig. 6. Tune for protons at extraction, by varying quadrupoles (table V). The bare machine value at injection is marked by the gray dot. 


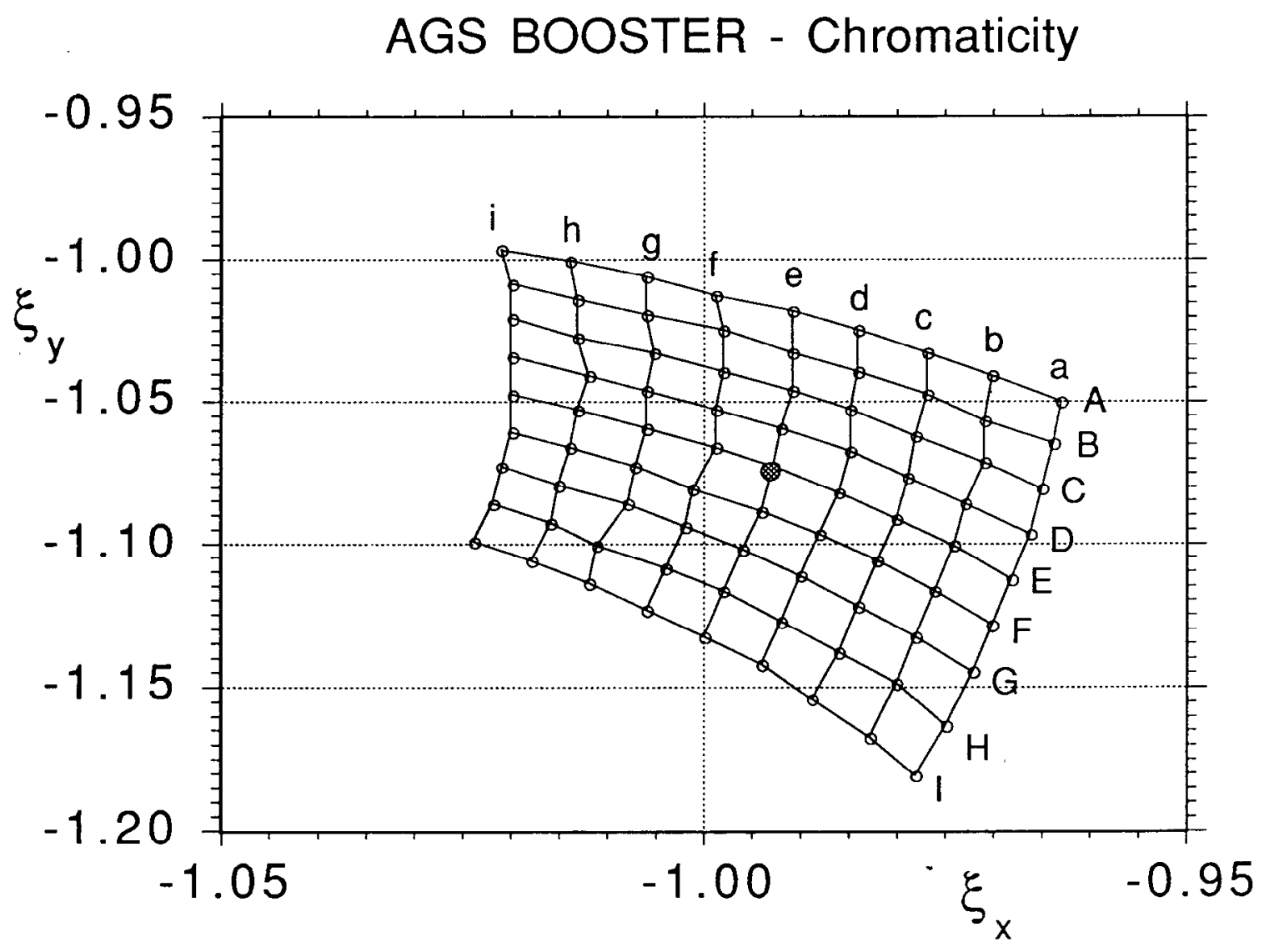

Fig. 7. Chromaticity for protons at extraction, by varying quadrupoles (table V). 


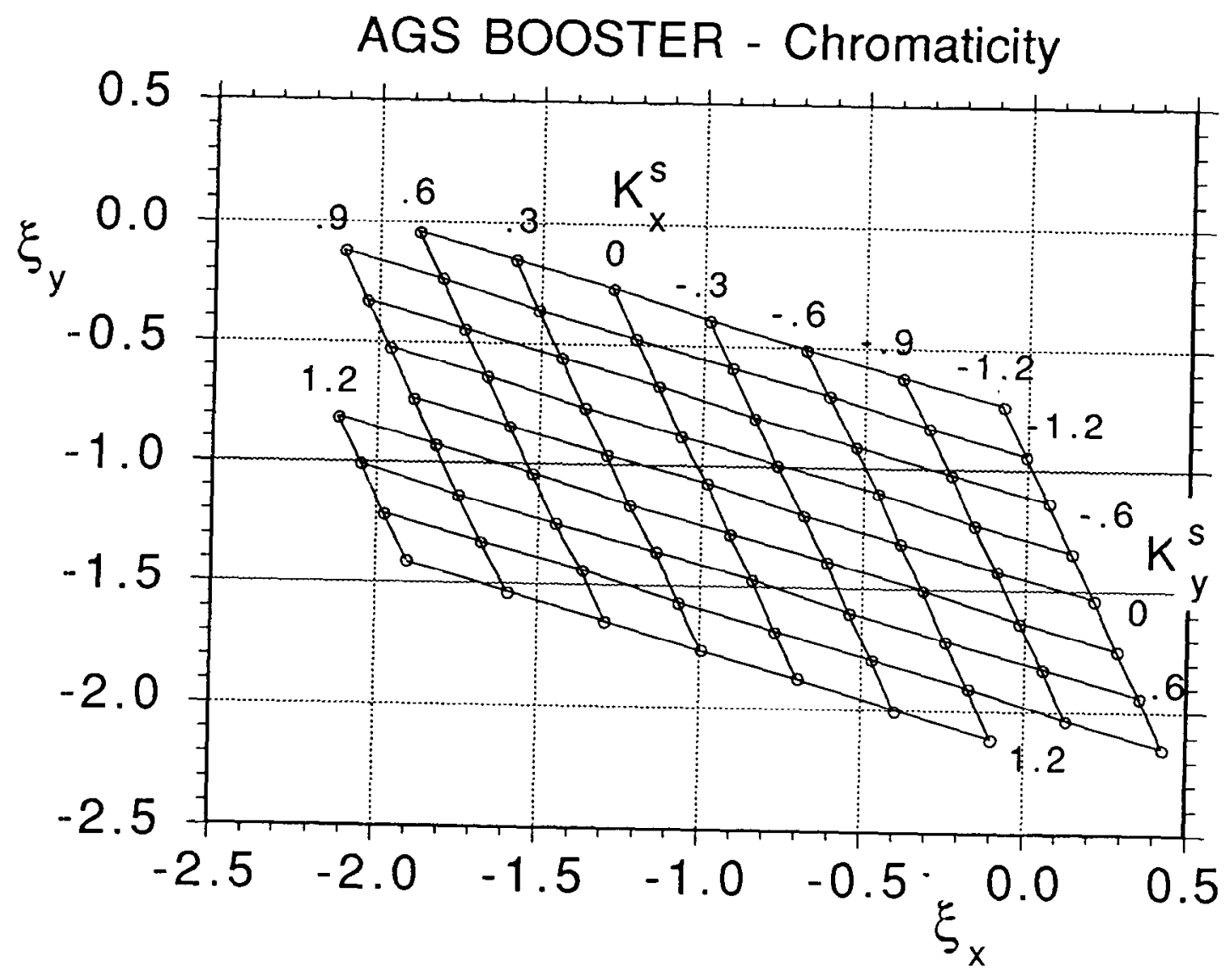

Fig. 8. Chromaticity for protons at injection, by varying sextupoles. Only the "main" out of three regions of values is shown. 